\title{
Search for solar energetic particle signals in the Mexico City neutron monitor database
}

\author{
B. Vargas-Cárdenas ${ }^{1}$ \\ J. F. Valdés-Galicia ${ }^{2}$ \\ ${ }^{1}$ Instituto de Geofísica, UNAM, México D.F., C.P. 04510. \\ email: bernardo@geofisica.unam.mx \\ ${ }^{2}$ Instituto de Geofísica, UNAM, México D.F., C.P. 04510. \\ email: valdes@geofisica.unam.mx
}

\begin{abstract}
We performed a search for ground level solar cosmic ray enhancements on the full five minute database of the Mexico City neutron monitor using wavelet filters and two different statistical tests. We present a detailed analysis of the time series of November 2, 1992, where we found a previously unreported increment matching the onset time of the impulsive phase of the Ground Level Enhancement No. 54, thus providing evidence of an effective detection of high energy solar cosmic rays. This technique may help to find still undiscovered GLE signals in the Worldwide Neutron Monitor Database, to refine GLE spectra and, probably, to find a relationship between the latter and the solar cycle.
\end{abstract}

Keywords. Ground level enhancements, solar energetic particles

\section{Introduction}

There is an increasing interest in evaluating the sun's maximum capacity to accelerate particles. This has motivated different studies with data from high cutoff rigidity neutron monitors (Debrunner et al. 1997; Karapetyan 2008; Beisembaev et al. 2009; Chilingarian 2009). Therefore, we decided to search for GLE signals in the whole database of Mexico City neutron monitor (http://132.248.105.25/). Due to the site's high cutoff rigidity $(8.27 \mathrm{GV})$ and high median response energy of the instrument to solar particles (10.7 $\mathrm{GeV}$ ), any such detection would provide evidence for the production of high energy particles by solar eruptive phenomena. We found only one signal on November 2, 1992 , whose significance could be validated by two different statistical tests, namely, Student's test (Karapetyan 2008; Beisembaev et al. 2009) and extremum statistics test (Chapman, Rowland \& Watkins 2002; Chilingarian 2009). Some authors (Shea \& Smart 2008; Belov, Eroshenko \& Kryakunova 2009) have studied the distribution of Ground Level Enhancements (GLE's) along the solar cycle, but it's not clear what is the relationship between sunspot numbers and GLE frequency, intensity or spectra.

\section{Data and Qualitative Analysis}

GOES-7 satellite registered an X9.0 flare beginning at 2:31 with a peak at 3:08 and ending at 3:28 UT of November 2 (Fig. 1). There were two coronal mass ejections associated with this flare; one starting at 2:53 with a type II radio burst and the other at 3:02 UT with a type IV burst. Although there was no sudden commencement, the Kp index increased to a value of 5.5, and a sudden ionospheric disturbance of importance 3 and a widespread index equal to 5 started at 2:32, peaked at 3:04 and ended at 9:40 UT. 
The direction of the IMF at 3:40 UT was $41^{\circ}$, a little eastward from the nominal Parker spiral, favoring the asymptotic directions of North American stations. To validate our claimed detection of a GLE signal on event 54, we also analyzed pressure corrected five minute count rates of Calgary, Deep River, Goose Bay, Hermanus, Inuvik, LARC, Lomnicky Stit, Oulu, Potchefstroom, SANAE and Tsumeb neutron monitors, as suggested by the Australian Antarctic Data Center (http://data.aad.gov.au/aadc/gle/); all these data were downloaded from the FTP site of IZMIRAN (ftp://cr0.izmiran.rssi.ru/). Dates and onset times of the events were taken from Miroshnichenko \& Pérez-Peraza (2008), and Shea et al. (1995). We used the baseline intervals suggested by the Bartol Research Institute (http://neutronm.bartol.udel.edu/). Solar data were taken from Solar Geophysical Data 580 and 581. All these neutron monitor data show the signature of the gradual phase of the event, beginning approximately at 4:00 and ending about 9:00 UT, but Deep River and Goose Bay show signs of the impulsive phase.

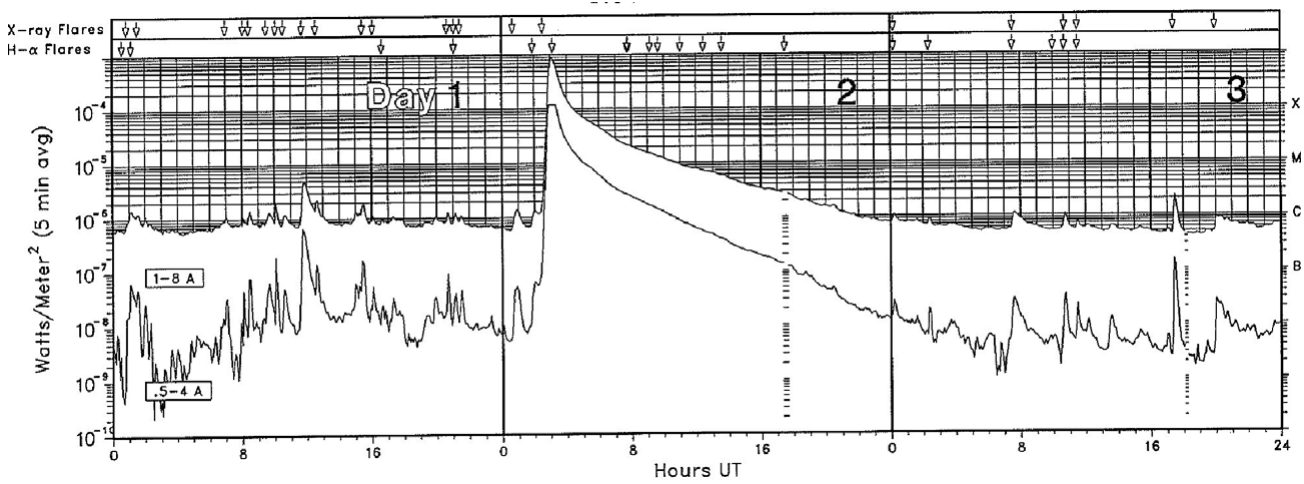

Figure 1. GOES-7 satellite hard and soft X ray data for November 1 to 3, 1992 taken from Solar Geophysical Data.

\section{Results}

Fig. 2 is a plot of Deep River and Goose Bay data flattened by wavelet based Daubechies filters (Daubechies 1988, 1993); both the impulsive and the gradual phases of the event are clearly distinguished. Deep River shows an increase in its count rates of $1.2 \%$ at 3:35 UT while Goose Bay has a 1.4\% increase 3:50 UT; this corresponds to the impulsive phase of the event.
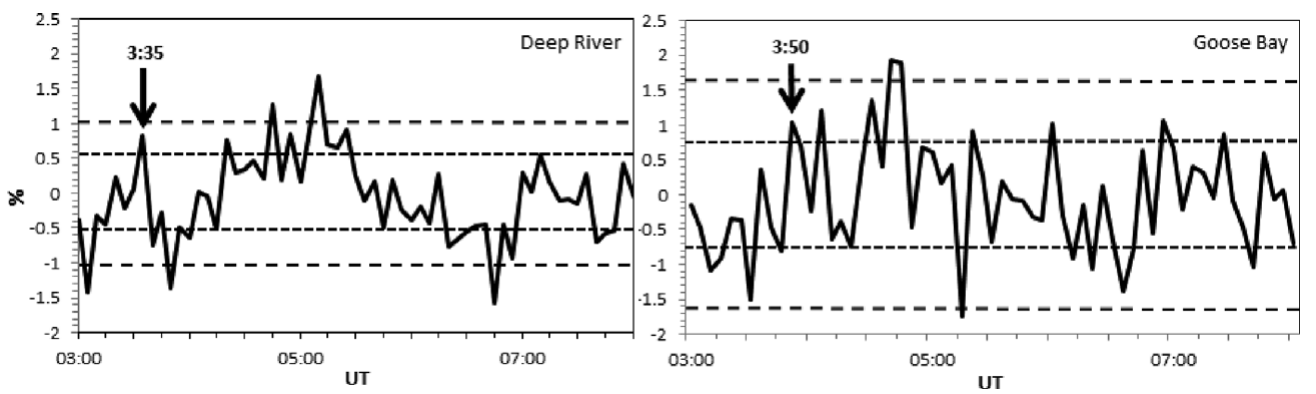

Figure 2. Flattened Deep River (left) and Goose Bay (right) neutron monitors data for November 2, 1992. Dashed lines indicate $\sigma$ and $2 \sigma$ thresholds. The two components of the event are evident on both plots. 
A second increase is observed between 4:30 and 6:30 UT, approximately; this is the signature of the gradual phase. Mexico City neutron monitor did not see the lower energy gradual phase, but it shows a $2.1 \%$ increase at 3:45 UT falling above the $2 \sigma$ threshold (Fig. 3), which should correspond to the impulsive phase. The difference in the timing of this peak between the three mentioned stations could be due to a high anisotropy in the high energy particle flux, but their coincidence in recording the event may be explained by the location of their corresponding asymptotic cones of acceptance, which are close to each other (Smart, Shea \& Flckiger 2000).

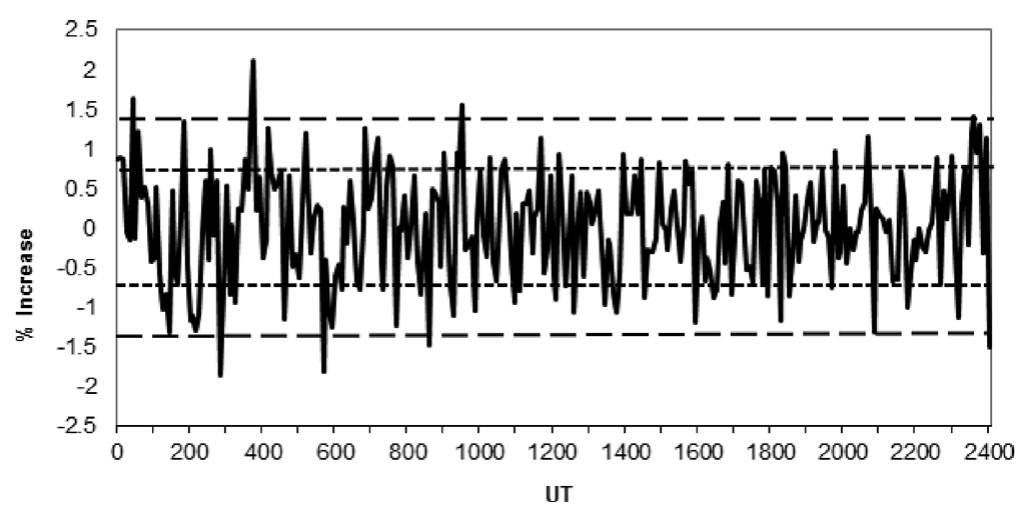

Figure 3. Mexico City data for November 2, 1992. Dashed lines represent $\sigma$ and $2 \sigma$ thresholds. The $2.1 \%$ increment observed at 3:45 UT corresponds to the onset time of GLE 54 .

Mexico City's cone of acceptance had a better connection with the IMF direction, hence the higher value of its increase in the count rates, irrespective of the higher cut off rigidity of the station. Fig. 3 represents Mexico City's time series corresponding to November 2, 1992, also flattened by Daubechies filters.

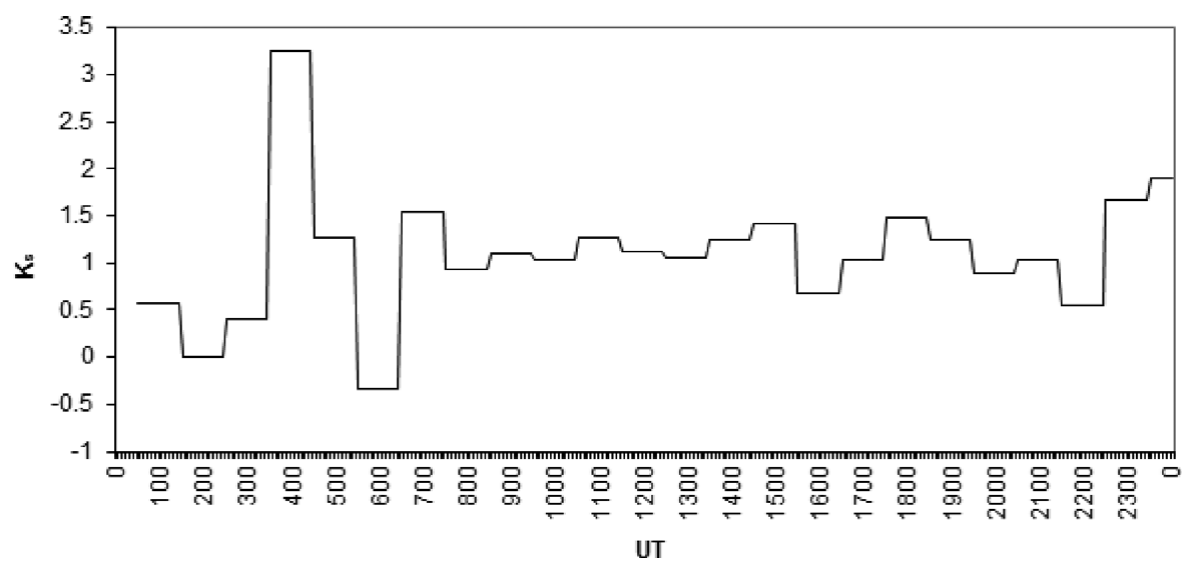

Figure 4. One hour Student's test for the Mexico City November 2, 1992 time series showing the significance of the 3:45 increment. 
The 2.1\% increment seen at 3:45 UT matches the reported onset time of GLE 54 and exceeds in 0.75 units the $2 \sigma$ threshold. This is the only positive peak that exceeds the $2 \sigma$ limit in the relevant time period. This result, together with the Goose Bay and Deep River flattened time series (Fig. 2) encouraged us to do the statistical analysis explained in Section 3. These could only be applied to Mexico City data as it is the only station without any trace of the gradual phase of the event. Fig. 4 shows Student's test for this time series. For the period including the peak at 3:45 UT we get a statistical significance of $99.8 \%$. Fig. 5 shows the plot of normalized residuals of the Mexico City neutron monitor data.

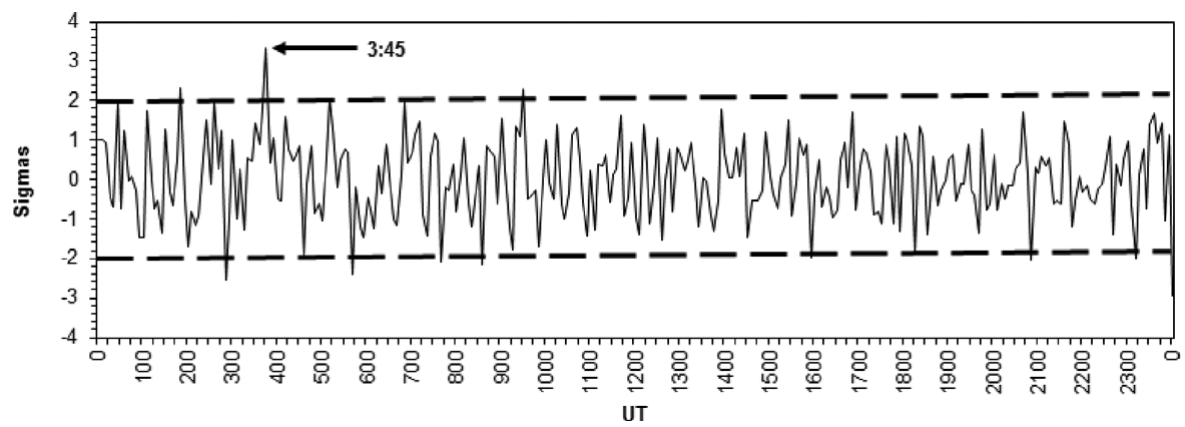

Figure 5. Residuals for the November 2, 1992 time series showing the significance of the 3:45 increment according to the extremum statistics test. Dashed lines represent the $2 \sigma$ threshold.

The residual corresponding to $3: 45$ UT is clearly standing out well above the $2 \sigma$ level and no other residual falls above it. The extremum statistics test gives a statistical significance of $96 \%$ for this peak. This is thus further evidence that the register in question might not correspond to a fluctuation of the isotropic galactic cosmic ray flux. As a consequence of the statistical tests performed to the Mexico City neutron monitor data during November 2, 1992, we can accept the hypothesis that we have found an additional cosmic ray signal superimposed to the galactic cosmic ray intensity with a statistical confidence that is beyond $96 \%$. As there is no other compatible source, this signal should correspond to particles produced during the impulsive phase of GLE 54. The instrument's mean counting rate of 76,470 counts/hour $=6,373$ counts $/ 5$ min with a corresponding standard deviation of $0.88 \%$ are an additional validation of the statistical results presented in this work. We calculate that under such disturbed geomagnetic conditions, the cutoff rigidity of the Mexico City site got lowered to a value of $7.5 \mathrm{GV}$; likewise, we have computed the median response energy of the instrument for the combined galactic and solar spectrum, using the upper limit spectum for protons (Miroshnichenko \& Pérez-Peraza 2008) and got a value of $10.7 \mathrm{GV}$. Thus, we can infer that the Sun emitted particles with energies of at least 7.5 and most probably $10.7 \mathrm{GeV}$ during the X9.0 flare that occurred on November 2,1992 .

\section{Conclusions}

We found evidence of the detection of solar particles during the event on 2 November 1992 (GLE 54) by the Mexico City neutron monitor, validated by two independent statistical tests, supporting the hypothesis that particles with energies around $10 \mathrm{GeV}$ were produced by the Sun. Mexico City data, as well as those from other middle and low latitude stations, can contribute substantially to refine solar energetic particle spectra 
and therefore to improve the corresponding acceleration models. Furthermore, they may help to evaluate the solar capability to produce high energy protons. In particular, these results may help to refine the upper limit spectrum for protons (Miroshnichenko 1996; Miroshnichenko and Pérez-Peraza 2008). Also, a more accurate knowledge of GLE spectra could lead to find a relationship with the solar cycle. Finally, we conjecture that there may be more solar particle event signals still undiscovered in the Worldwide Neutron Monitor Network's database that require refined statistical analysis to be revealed.

\section{References}

Beisembaev, R., Dorbzhev, V., Dryn, E., \& Kryakunova, O. 2009, Adv. in Sp. Res., 43, 509

Belov, A. V., Eroshenko, E. A., Kryakunova, O. N., Kurt, V. G., \& Yanke, V. G. 2009, Proceedings of the 31st Int. Cosmic Ray Conf., Lodz

Chapman, S. C., Rowlands, G., \& Watkins, N. W. 2002, Nuclear Processes in Geophysics, 9, 409

Chilingarian, A. 2009, Adv. in Sp. Res., 43, 702

Daubechies, I. 1988, Communications on Pure and Applied Mathematics, 41, 909

Daubechies, I. 1993, SIAM Journal on Mathematical Analysis, 24, 499

Debrunner, H., Lockwood, J. A., Barat, C., Btikofer, R., Dezalay, J. P., Flckiger, E., Kuznetsov, A., Ryan, J. M., Sunyaev, R., Terekhov, O. V., Trottet, G., \& Vilmer, N. 1997, Astrophys. J., 479,997

Karapetyan, G. G. 2008, Astroparticle Physics, 30(5), 234

Miroshnichenko, L. I. 1996, Radiation Measurements, 26(3), 421

Miroshnichenko, L. I. \& Pérez-Peraza, J. A. 2008, International Journal of Modern Physics A, $23(1), 1$

Shea, M. A., Smart, D. F., Gentile, L. C., \& Campbell, J. M. 1995, in: N. Iucci, E. Lamanna (Eds.), Proceedings of the 24nd Int. Cosmic Ray Conf. (International Union of Pure and Applied Physics, Rome), Vol. 4, 244

Shea, M. A. \& Smart, D. F. 2008, in: R. Caballero, J. C. D'Olivo, G. Medina-Tanco, L. Nellen, F. A. Snchez, J. F. Valds-Galicia (eds.), Proceedings of the 30th Int. Cosmic Ray Conf. (Universidad Nacional Autnoma de Mxico, Mexico City), Vol. 1, 261

Smart, D. F., Shea, M. A., \& and Flckiger, E. O. 2000, Space Science Reviews 93, 305

Solar Geophysical Data No. 580, Part I 1992, National Geophysical Data Center, Boulder, December 1992

Solar Geophysical Data No. 581, Part II 1993, National Geophysical Data Center, Boulder, January 1993

\section{Discussion}

JAnET Luhmann: Do you have any doubts to see a solar cycle dependence of the GLEs? Wouldn't you expect it to depend on the existence of very fast CME shocks in the corona which generally happen during active times?

Bernardo VARgAs: We believe these are flare-accelerated particles and not CMEaccelerated particles, since shocks cannot accelerate particles to these energies; I mean, nobody has explained how a shock could accelerate particles to these high energies in an efficient manner. Don't forget these particles are relativistic. 